\title{
Long exposure to mature ooplasm can alter DNA methylation at imprinted loci in non-growing oocytes but not in prospermatogonia
}

\author{
Yayoi Obata, Takuya Wakai, Satoshi Hara and Tomohiro Kono \\ Department of Bioscience, Tokyo University of Agriculture, 1-1-1 Sakuragaoka, Setagaya-ku, Tokyo 156-8502, Japan \\ Correspondence should be addressed to Y Obata; Email: y1obata@nodai.ac.jp
}

\begin{abstract}
DNA methylation imprints that are established in spermatogenesis and oogenesis are essential for functional gametes. However, the mechanisms underlying gamete-specific imprinting remain unclear. In this study, we investigated whether male and female gametes derived from newborn mice are epigenetically plastic and whether DNA methylation imprints are influenced by the niche surrounding the nuclei of the gametes. When prospermatogonia possessing sperm-specific DNA methylation imprints were fused with enucleated fully grown oocytes and exposed to the ooplasm for 5-6 days, the DNA methylation status of the reconstituted oocytes remained identical to that of prospermatogonia for all the imprinted regions analysed. These results suggest that the imprinting status of prospermatogonia is stable and that the epigenome of prospermatogonia loses sexual plasticity. By contrast, when non-growing oocytes lacking oocytespecific DNA methylation imprints were fused with enucleated fully grown oocytes and the reconstituted oocytes were then cultured for 5-6 days, the Igf2r, Kcnq1ot1 and, unexpectedly, H19/Igf2 differentially methylated regions (DMRs) were methylated. Methylation imprints were entirely absent in oocytes derived from 5-day-old mice, and H19/Igf2 DMR is usually methylated only in spermatogenesis. These findings indicate that in the nuclei of non-growing oocytes the chromatin conformation changes and becomes permissive to DNA methyltransferases in some DMRs and that mechanisms for maintaining non-methylated status at the H19/Igf2 DMR are lost upon long exposure to mature ooplasm.
\end{abstract}

Reproduction (2014) 147 H1-H6

\section{Introduction}

DNA methylation imprints that are established in spermatogenesis and oogenesis are maintained after fertilisation, which results in parental-origin-specific gene expression in the somatic cell lineage. By contrast, in the germ cell lineage, parental-origin-specific DNA methylation imprints are erased and gametes acquire new imprints according to their own sex (Ferguson-Smith 2011, Obata 2011).

It has been reported that the maintenance of allelespecific DNA methylation is required for protection against DNA demethylation by pluripotency-associated protein 3 (DPPA3; Nakamura et al. 2007, 2012), maintenance of DNA methylation by DNA methyltransferase 1 (DNMT1; Li et al. 1993), and maintenance of the unmethylated status of differentially methylated regions (DMRs) by CTCF (Schoenherr et al. 2003, Szabo et al. 2004). During the erasure of DNA methylation imprints in the germ cell lineage, maintenance mechanisms for DNA methylation imprints must be disrupted, although the precise nature of such disruption remains unknown.
DNMT3A and DNMT3L are essential for the acquisition of DNA methylation imprints in both male and female gametes (Bourc'his et al. 2001, Hata et al. 2002, Kaneda et al. 2004). Sperm-specific DNA methylation imprints are established during the mitotic arrest of prospermatogonia in foetal testes (Davis et al. 2000, Hiura et al. 2007), whereas oocyte-specific DNA methylation imprints are established during oocyte growth, and oocytes possessing all imprints first appear in the peri-pubertal stage (Lucifero et al. 2004, Hiura et al. 2006).

We have demonstrated that oocytes obtained from sex-reversed $X Y$ female B6. $Y^{\mathrm{TIR}}$ mice do not naturally develop beyond the four-cell stage (Villemure et al. 2007), but can develop to term after cytoplasmic exchange with oocytes from XX females, IVF and embryo transfer (Obata et al. 2008). Therefore, the nuclei of oocytes from XY females are able to acquire oocytespecific imprints. However, unless sex reversal occurs, B6. $Y^{\mathrm{TIR}}$ mice are fully fertile males. Thus, germ cells are epigenetically bipotential in the early stage and can acquire either sperm- or oocyte-specific imprints independent of their genetic sex. Sex reversal in B6. $Y^{\mathrm{TIR}}$ mice 
is caused by the delayed and decreased expression of Sry in gonadal somatic cells (Park et al. 2011), which suggests that the niche surrounding germ cells controls the sex of the gametes, including their imprinting.

In this study, to investigate whether epigenetic plasticity is maintained in male and female gametes derived from newborn mice and whether DNA methylation imprints are influenced by the niche surrounding the nuclei of gametes, prospermatogonia possessing sperm-specific DNA methylation imprints and nongrowing oocytes lacking oocyte-specific DNA methylation imprints were fused with fully grown oocytes respectively. The reconstituted oocytes were cultured for 5-6 days and then subjected to DNA methylation analysis of imprinted genes.

\section{Materials and methods Animals}

All the procedures used in this study were reviewed and approved by the Tokyo University of Agriculture Institutional Animal Care and Use Committee and were carried out in accordance with the Guidelines for Proper Conduct of Animal Experiments, as established by the Science Council of Japan. BDF1 (C57BL/6N×DBA/2; CLEA Japan, Tokyo, Japan) hybrid mice were used for all the experiments. JF1 mice (Mus musculus molossinus) were used only in the polymorphic analysis of DNA methylation (Koide et al. 1998).

\section{Prospermatogonia and oocytes}

Prospermatogonia and non-growing oocytes were collected from newborn mice ( $0-2$ days postpartum). Testes and ovaries were treated with $0.1 \%$ collagenase (Wako, Osaka, Japan) in BSA-free $\mathrm{M} 2$ medium for $20 \mathrm{~min}$ at $37{ }^{\circ} \mathrm{C}$ and were then treated with $0.05 \%$ trypsin-0.53 mM EDTA (Gibco, Life Technologies Japan) in PBS for $15 \mathrm{~min}$ at $37^{\circ} \mathrm{C}$. The cells of the testes and ovaries were then dispersed and suspended by pipetting in $\mathrm{M} 2$ medium containing $10 \mu \mathrm{g} / \mathrm{ml}$ cytochalasin B (Sigma-Aldrich Japan), $100 \mathrm{ng} / \mathrm{ml}$ demecolcine (Wako) and $240 \mu \mathrm{M}$ dibutyryl cAMP (Sigma-Aldrich Japan).

Cumulus-oocyte complexes (COCs) were collected from the antral follicle of the ovaries of adult female mice 40-42 h after the injection of $5 \mathrm{IU}$ of equine chorionic gonadotrophin (Serotropin; ASKA Pharmaceutical Company, Tokyo, Japan). Fully grown oocytes at the germinal vesicle (GV) stage were denuded by pipetting. Cumulus cells were used for the reconstruction of COCs.

\section{Fusion of ooplasm and gametes}

Fusion experiments were conducted in M2 medium containing $10 \mu \mathrm{g} / \mathrm{ml}$ cytochalasin B, $100 \mathrm{ng} / \mathrm{ml}$ demecolcine and $240 \mu \mathrm{M}$ dibutyryl CAMP using a nuclear transfer technique. Prospermatogonia and non-growing oocytes were fused with enucleated or intact fully grown oocytes respectively using an inactivated Sendai virus (2700 haemagglutinating activity (units/ml)). As a control, the GVs of fully grown oocytes were fused with enucleated fully grown oocytes. After fusion, the zona pellucida was removed mechanically using a micromanipulator with a glass needle. To reconstitute COCs, these oocytes were co-cultured with cumulus cells in $\alpha$-MEM (Gibco, 12000-022; Life Technologies Japan) supplemented with $2.2 \mathrm{~g} / \mathrm{l} \mathrm{NaHCO}, 3.5 \mathrm{~g} / \mathrm{l}$ glucose, $240 \mu \mathrm{M}$ dibutyryl cAMP and $5 \%$ FBS in $5 \% \mathrm{CO}_{2}, 5 \% \mathrm{O}_{2}$ and $90 \% \mathrm{~N}_{2}$ at $37{ }^{\circ} \mathrm{C}$ for 5 days. Approximately 25 oocytes were co-cultured with cumulus cells isolated from 60 COCs in a $100 \mu$ drop of medium covered with paraffin oil, and half of the medium was exchanged every 2 days (Fig. 1).

\section{DNA methylation analysis}

Prior to lysis, reconstituted COCs were treated with $0.05 \%$ trypsin-0.53 mM EDTA in PBS, and cumulus cells were completely removed by pipetting. Approximately 200 intact gametes or reconstituted oocytes were pooled in $18 \mu \mathrm{l}$ of lysis buffer ( $1 \%$ SDS, $5 \mu \mathrm{g}$ of proteinase $\mathrm{K}$ and $2 \mu \mathrm{g}$ of yeast tRNA) and then incubated at $37^{\circ} \mathrm{C}$ for $1 \mathrm{~h}$ to extract DNA. We used the CpGenome DNA Modification Kit (Millipore, Billerica, MA, USA) for sodium bisulphite conversion of DNA. DNA methylation was assessed at a DMR in each of the five imprinted regions: Igf2r, Kcnq1ot1, Mest, H19/lgf2 and Dlk1/Gt/2. Primers and PCR conditions are summarised in Supplementary Table 2, see section on supplementary data given at the end of this article. Bisulphite-modified DNA from 40 to 80 gametes was subjected to PCR for each gene. PCR products were cloned into a T Easy vector (pGEM T Easy vector; Promega) and then sequenced using an Applied Biosystems sequencing system (ABI PRISM 3100, Tokyo, Japan). Three pools of DNAs were analysed independently. Differences in methylation levels were assessed using the Mann-Whitney $U$ test.

\section{Immunostaining}

For immunostaining of DNMT3A and DNMT3L (Sakai et al. 2004, Hirasawa et al. 2008), reconstituted oocytes were fixed
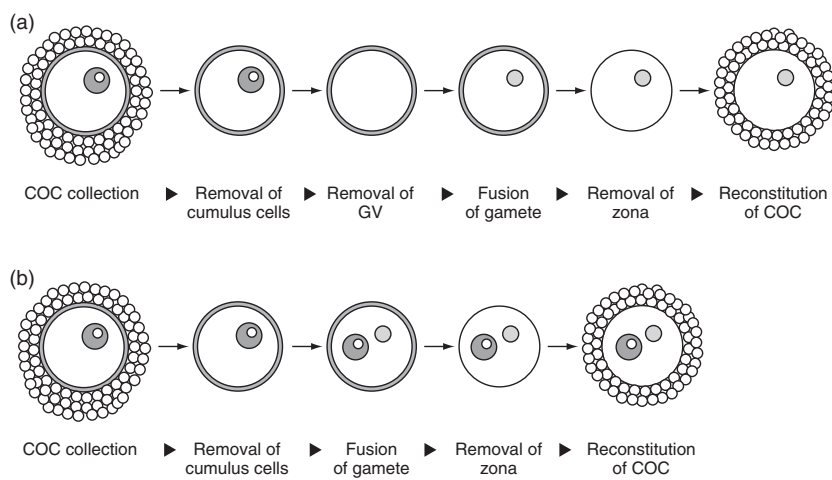

Figure 1 Schematic diagram of COC reconstitution. (a) GV is removed from a fully grown oocyte before fusion. The prospermatogonium or non-growing oocyte is fused with the enucleated oocyte. (b) GV is not removed from a fully grown oocyte before fusion. The prospermatogonium or non-growing oocyte derived from a JF1 newborn mouse is fused with the intact GV oocyte from a BDF1 adult mouse for polymorphic analysis of DNA methylation. 
lgf2r DMR

(a)

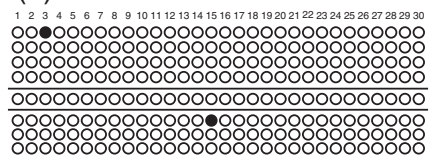

\section{Lit1 DMR}

(d)

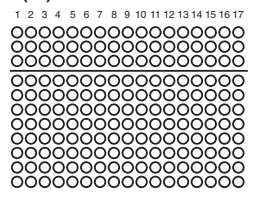

H19/lgf2 DMR

(g)

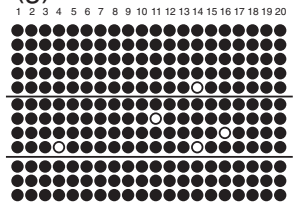

Igf2r DMR

(j)

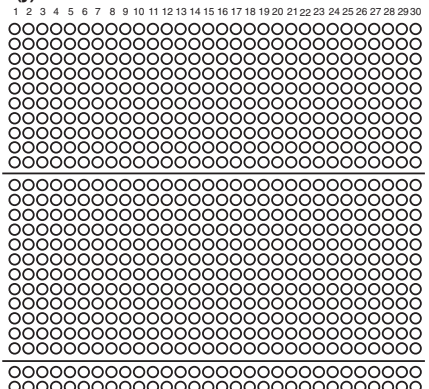

it1 DMR

(m)

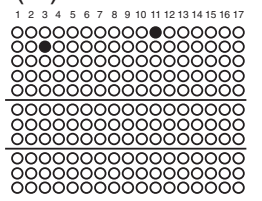

H19/lgf2 DMR

(p)

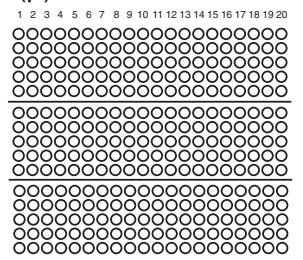

(b)

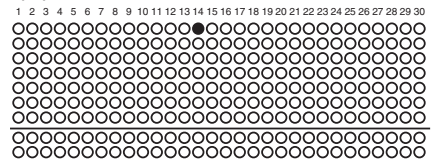

(e)

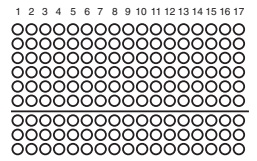

(h)

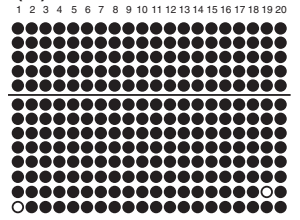

(k)

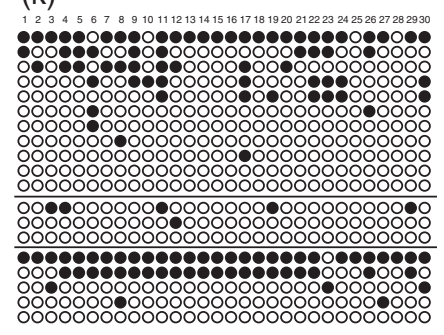

(c)

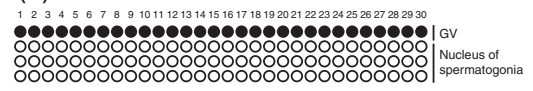

(f)

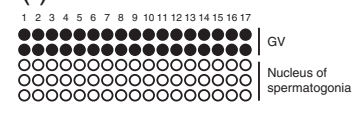

(i)

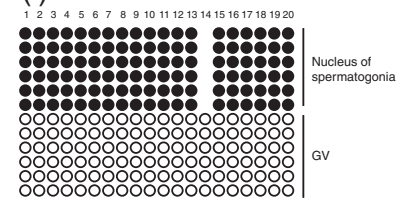

(l)

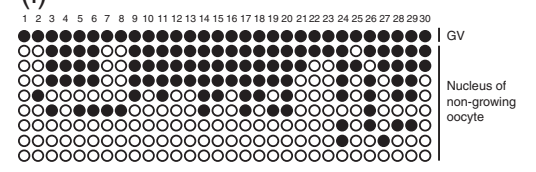

(0)

(n)
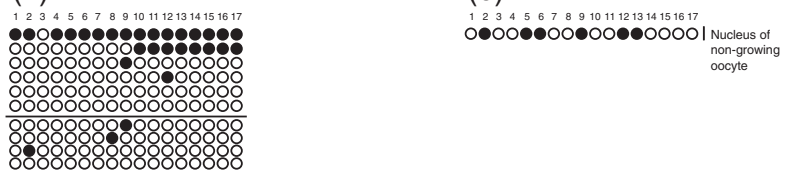

(r)

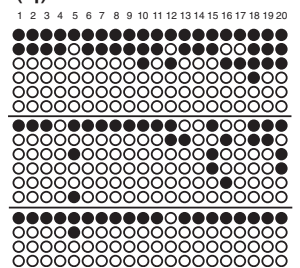

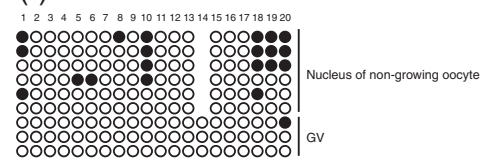

Figure 2 DNA methylation status in reconstituted oocytes after 5-6 days of culture. (a, $d$ and g) DNA methylation status of prospermatogonia. (b, e and h) DNA methylation status of oocytes containing nuclei derived from prospermatogonia after 5-6 days of culture. (c, $f$ and i) DNA methylation status of oocytes containing nuclei derived from prospermatogonia and GV after 5-6 days of culture. (j, $m$ and p) DNA methylation status of non-growing oocytes. ( $k, n$ and $q$ ) DNA methylation status of oocytes containing nuclei derived from non-growing oocytes after 5-6 days of culture. ( $($, o and $r$ ) DNA methylation status of oocytes containing nuclei derived from non-growing and fully grown oocytes after 5-6 days of culture. Filled circle, methylated cytosine; open circle, unmethylated cytosine. A line of circles represents a single DNA strand. Each pool of oocytes is distinguishable by the horizontal lines. 
and permeabilised with $2 \%$ paraformaldehyde and $0.2 \%$ Triton $\mathrm{X}-100$ in PBS for $1 \mathrm{~h}$ at room temperature. Then, the oocytes were incubated in PBS supplemented with 3\% BSA for $1 \mathrm{~h}$ at room temperature. After blocking, the oocytes were incubated with mouse anti-DNMT3L polyclonal antibody (gifted from Shoji Tajima; dilution rate $1: 100$ ) overnight at $4{ }^{\circ} \mathrm{C}$ followed by incubation with DNMT3A MAB (IMGENEX, San Diego, CA, USA; IMG-268A CA; dilution rate 1:300). The oocytes were washed with PBS containing $0.1 \%$ Triton X-100 and 1\% BSA for 20 min each. Subsequently, the oocytes were incubated with Alexa488-conjugated anti-mouse IgG for DNMT3A detection (Molecular Probes; dilution rate 1:500) and Alexa568-conjugated anti-rabbit IgG for DNMT3L detection (Life Technologies, Grand Island, NY, USA; dilution rate 1:500). Nuclei were counterstained using DAPI. The oocytes were observed using a confocal laser scanning microscope (LSM710, Carl Zeiss Japan, Tokyo, Japan).

\section{Results and discussion}

\section{Fusion of ooplasm and gametes}

To determine whether DNA methylation imprints are influenced by the niche surrounding the nuclei of the gametes, we conducted fusion experiments using nuclear transfer technique and COC reconstitution for long exposure of nuclei derived from gametes in the cytoplasm of fully grown oocytes (Fig. 1).

Prior to the fusion experiments, cumulus cells should be removed from GV oocytes. However, denuded oocytes were not viable within $48 \mathrm{~h}$. Therefore, denuded oocytes were co-cultured with cumulus cells after the removal of the zona. Cumulus cells adhered tightly to zona-free oocytes, and the resultant oocytes had a healthy appearance (Supplementary Figure 1, see section on supplementary data given at the end of this article and Supplementary Table 1). When 7 or more days had elapsed, some oocytes were denuded naturally. Hence, we assessed the DNA methylation status within 5-6 days of the initiation of culture.

\section{Imprinting status of nuclei derived from prospermatogonia after long exposure to ooplasm}

As has been reported previously (Davis et al. 2000, Hiura et al. 2007), H19/lgf2 and D/k1/Gtl2 intergenic (IG) DMRs were fully methylated, whereas Igf2r,
Kcnq1ot1 and Mest DMRs were not methylated in prospermatogonia derived from newborn mice (Fig. 2 and Table 1). Prospermatogonia were fused with enucleated or intact fully grown oocytes, and these oocytes were then cultured with cumulus cells for 5-6 days. However, the DNA methylation status of the reconstituted oocytes was not altered; it remained identical to that of prospermatogonia in all the analysed regions (Fig. 2 and Table 1 ). Therefore, it is likely that the imprinting status of prospermatogonia is stable and that the epigenome of prospermatogonia loses sexual plasticity. However, Wang et al. (2012) reported that spermatogonial cells from 8-day-old mice differentiated into oocyte-like cells and that, surprisingly, spermspecific imprints were converted into oocyte-specific imprints over a culture period of 3 weeks. This appears to contradict our results; it is possible that spermatogonial stem cells cultured under certain conditions de-differentiated to primordial germ cell-like cells and the sperm-specific imprints were erased prior to development into oocyte-like cells.

\section{Imprinting status of nuclei derived from non-growing oocytes after long exposure to ooplasm}

In non-growing oocytes derived from newborn mice, H19/lgf2, DIk1/Gtl2 IG, Igf2r, Kcnq1ot1 and Mest DMRs were not methylated (Fig. 2 and Table 1). These nongrowing oocytes were fused with enucleated or intact fully grown oocytes, and fused oocytes were then cultured with cumulus cells. After 5-6 days of culture, Igf2 $r$ and Kcnq1 ot1 DMRs showed 0-98.6\% methylation in nuclei derived from non-growing oocytes. Furthermore, the H19/lgf2 DMR, which is hypermethylated only in spermatogenesis, was also methylated (Fig. 2). De novo DNA methylation at the DMRs analysed did not occur in oocytes derived from 5-day-old mice. As a control experiment, nuclei derived from fully grown oocytes were fused with enucleated fully grown oocytes and these oocytes were cultured with cumulus cells for 5-6 days. However, the H19/Igf2 DMR was not methylated (Table 1), which indicates that the DNA methylation of the H19/lgf2 DMR in nuclei derived from non-growing oocytes was caused by long exposure to the mature ooplasm niche rather than due to micromanipulation. These results suggest that in the nuclei of

Table 1 Rate of methylated CpG sites at each imprinted locus in reconstituted oocytes after 5-6 days of culture.

\begin{tabular}{|c|c|c|c|c|c|c|}
\hline \multirow[b]{2}{*}{ Donor nuclei } & \multicolumn{2}{|c|}{ Prospermatogonia } & \multicolumn{3}{|c|}{ Non-growing oocytes } & \multirow{2}{*}{$\begin{array}{c}\text { GV oocytes } \\
\text { Exposed }\end{array}$} \\
\hline & Intact & Exposed & Intact & Intact $^{\mathrm{a}}$ & Exposed & \\
\hline $\lg f 2 r$ & 0.8 & 0.3 & 0 & 0 & $27.7^{*}$ & 99.6 \\
\hline Kcnq1ot1 & 0 & 0 & 1.1 & 1.5 & $18.7^{*}$ & 97.1 \\
\hline Mest & 0 & 0.4 & 0.7 & 0.7 & 1.4 & 100 \\
\hline H19/Igf2 & 97.2 & 99.5 & 0 & 0.8 & $23.0^{*}$ & 0.8 \\
\hline Dlk1/Gtl2 & 100 & 99.1 & 0.5 & 0.3 & 1.7 & 0.5 \\
\hline
\end{tabular}

$* P<0.05$.

${ }^{a}$ Oocytes derived from 5-day-old mice. 
non-growing oocytes mechanisms for maintaining the unmethylated status are lost or that the chromatin conformation changes and becomes permissive to DNMTs in some DMRs upon exposure to the mature ooplasm niche.

To confirm that this methylation was induced by DNMT3A and DNMT3L, we carried out immunostaining in the oocytes fused with gametes (Fig. 3). Immediately after fusion, nuclei derived from prospermatogonia and non-growing oocytes were condensed, and DNMT3A and DNMT3L were weakly detected in
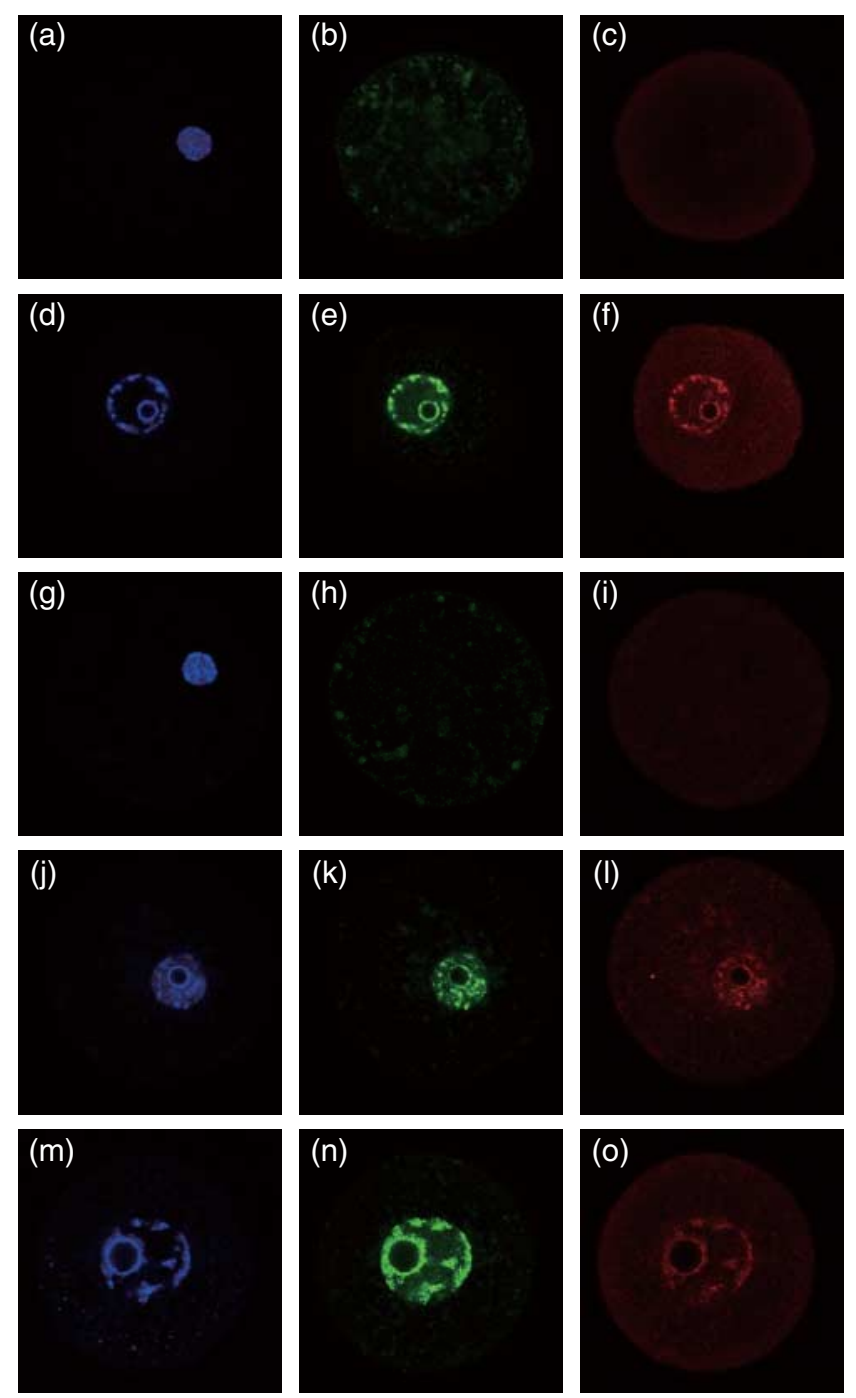

Figure 3 Immunostaining of reconstituted oocytes after 5 days of culture. $(a, b$ and $c)$ Oocyte containing nucleus derived from a prospermatogonium immediately after fusion. (d, e and f) Oocyte containing nucleus derived from a prospermatogonium after 5 days of culture. ( $g$, h and i) Oocyte containing nucleus derived from a non-growing oocyte immediately after fusion. (j, k and I) Oocyte containing nucleus derived from a non-growing oocyte after 5 days of culture. ( $\mathrm{m}, \mathrm{n}$ and o) GV oocyte. Blue, DAPI (nucleus); green, DNMT3A and red, DNMT3L. the nuclei of prospermatogonia but were absent in the nuclei of non-growing oocytes. Five days after the initiation of culture, the nuclei had swelled, and DNMT3A and DNMT3L were detected in both types of nuclear-transferred oocytes. Some nucleoli clearly appeared in the nucleus. It is not known whether DNMT3A and DNMT3L were translated from the mRNA of the recipient cytoplasm or from the newly synthesised mRNA of the donor nuclei in the present study. Regardless, the existence of DNMT3A and DNMT3L in the nuclei of non-growing oocytes must be related to the alteration in DNA methylation. By contrast, when nuclei derived from non-growing oocytes were exposed to the mature ooplasm niche, the DNA methylation status of Mest was not affected (Table 1). It is known that oocyte-specific DNA methylation imprints are established with gene-specific timing. Mest is a gene whose imprinting is established in the later stage of oocyte growth (Obata \& Kono 2002, Lucifero et al. 2004, Hiura et al. 2006). Whether DNA methylation is induced in nuclei derived from nongrowing oocytes by exposure to the mature ooplasm niche may depend on the mechanisms for the establishment of imprinting in each gene. Furthermore, in oocytes containing the nuclei of prospermatogonia, $\lg 2 \mathrm{r}$ and Kcnq1ot1 DMRs did not enable epigenetic alteration in spite of nuclear swelling and DNMT3A and DNMT3L reactivation. This finding indicates that oocyte-specific methylated regions are shielded from DNMT3A and DNMT3L in prospermatogonia even after long exposure to the ooplasm niche.

In mice, sexual dimorphism of germ cells is obvious in the embryonal gonads at 13.5 days postcoitum (dpc). Male germ cells show arrested mitosis, whereas female germ cells enter meiosis (McLaren 1991). Prior to the sex differentiation of germ cells, Sry and Sox9 are expressed in the somatic cells in the male gonad, which leads to testes cord formation and Sertoli and Leydig cell differentiation (Kashimada \& Koopman 2010). Adams \& McLaren (2002) demonstrated that the timing of entry into meiosis or arrest at mitosis in germ cells is governed by gonadal somatic cells until $12.5-13.5 \mathrm{dpc}$ and that germ cells have bipotential independent of their genetic sex until $11.5 \mathrm{dpc}$. From our results, it is unclear whether the timing of the commitment to mitosis/meiosis is concomitant with the timing of commitment to sperm/ oocyte-specific imprinting. However, it is intriguing that the H19/lgf2 DMR was methylated in nuclei derived from non-growing oocytes that had already entered into meiosis. It is known that CTCF binds to specific sites in the non-methylated H19/lgf2 DMR (Schoenherr et al. 2003, Szabo et al. 2004). CTCF knockdown during oogenesis induces the methylation of the H19/lgf2 DMR in oocytes (Fedoriw et al. 2004). Thus, it is likely that the maintenance of the non-methylated status of DMRs is important for establishing sperm- or oocyte-specific methylation imprints. 


\section{Supplementary data}

This is linked to the online version of the paper at http://dx.doi. org/10.1530/REP-13-0359.

\section{Declaration of interest}

The authors declare that there is no conflict of interest that could be perceived as prejudicing the impartiality of the research reported.

\section{Funding}

This work was supported by the Naito Foundation, grants-in-aid for scientific research 23616006 and 25114008, and MEXTSupported Program for the Strategic Research Foundation at Private Universities to Y Obata.

\section{Acknowledgements}

The authors thank Prof. Shoji Tajima (Osaka University) for providing the anti-DNMT3L antibody, Prof. Toshihiko Shiroishi (National Institute of Genetics) for providing the JF1 mouse strain, and Prof. Hisataka Iwata and Dr Hajime Matsubara (Tokyo University of Agriculture) for their helpful comments.

\section{References}

Adams IR \& McLaren A 2002 Sexually dimorphic development of mouse primordial germ cells: switching from oogenesis to spermatogenesis. Development 129 1155-1164.

Bourc'his D, Xu GL, Lin CS, Bollman B \& Bestor TH 2001 Dnmt3L and the establishment of maternal genomic imprints. Science 294 2536-2539. (doi:10.1126/science.1065848)

Davis TL, Yang GJ, McCarrey JR \& Bartolomei MS 2000 The H19 methylation imprint is erased and re-established differentially on the parental alleles during male germ cell development. Human Molecular Genetics 9 2885-2894. (doi:10.1093/hmg/9.19.2885)

Fedoriw AM, Stein P, Svoboda P, Schultz RM \& Bartolomei MS 2004 Transgenic RNAi reveals essential function for CTCF in H19 gene imprinting. Science 303 238-240. (doi:10.1126/science.1090934)

Ferguson-Smith AC 2011 Genomic imprinting: the emergence of an epigenetic paradigm. Nature Reviews. Genetics 12 565-575. (doi:10.1038/ nrg3032)

Hata K, Okano M, Lei H \& Li E 2002 Dnmt3L cooperates with the Dnmt3 family of de novo DNA methyltransferases to establish maternal imprints in mice. Development 129 1983-1993.

Hirasawa R, Chiba H, Kaneda M, Tajima S, Li E, Jaenisch R \& Sasaki H 2008 Maternal and zygotic Dnmt1 are necessary and sufficient for the maintenance of DNA methylation imprints during preimplantation development. Genes and Development 22 1607-1616. (doi:10.1101/ gad.1667008)

Hiura H, Obata Y, Komiyama J, Shirai M \& Kono T 2006 Oocyte growthdependent progression of maternal imprinting in mice. Genes to Cells $\mathbf{1 1}$ 353-361. (doi:10.1111/j.1365-2443.2006.00943.x)

Hiura H, Komiyama J, Shirai M, Obata Y, Ogawa H \& Kono T 2007 DNA methylation imprints on the IG-DMR of the Dlk1-Gt12 domain in mouse male germline. FEBS Letters 581 1255-1260. (doi:10.1016/j.febslet. 2007.02.034)
Kaneda M, Okano M, Hata K, Sado T, Tsujimoto N, Li E \& Sasaki H 2004 Essential role for de novo DNA methyltransferase Dnmt3a in paternal and maternal imprinting. Nature 429 900-903. (doi:10.1038/ nature02633)

Kashimada K \& Koopman P 2010 Sry: the master switch in mammalian sex determination. Development 137 3921-3930. (doi:10.1242/dev. 048983)

Koide T, Moriwaki K, Uchida K, Mita A, Sagai T, Yonekawa H, Katoh H, Miyashita N, Tsuchiya K, Nielsen TJ et al. 1998 A new inbred strain JF1 established from Japanese fancy mouse carrying the classic piebald allele. Mammalian Genome 9 15-19. (doi:10.1007/s003359900672)

Li E, Beard C \& Jaenisch R 1993 Role for DNA methylation in genomic imprinting. Nature 366 362-365. (doi:10.1038/366362a0)

Lucifero D, Mann MR, Bartolomei MS \& Trasler JM 2004 Gene-specific timing and epigenetic memory in oocyte imprinting. Human Molecular Genetics 13 839-849. (doi:10.1093/hmg/ddh104)

McLaren A 1991 Development of the mammalian gonad: the fate of the supporting cell lineage. BioEssays 13 151-156. (doi:10.1002/bies. 950130402)

Nakamura T, Arai $Y$, Umehara $H$, Masuhara $M$, Kimura T, Taniguchi $H$, Sekimoto T, Ikawa M, Yoneda Y, Okabe M et al. 2007 PGC7/Stella protects against DNA demethylation in early embryogenesis. Nature Cell Biology 9 64-71. (doi:10.1038/ncb1519)

Nakamura T, Liu YJ, Nakashima H, Umehara H, Inoue K, Matoba S, Tachibana M, Ogura A, Shinkai Y \& Nakano T 2012 PGC7 binds histone $\mathrm{H} 3 \mathrm{~K} 9 \mathrm{me} 2$ to protect against conversion of $5 \mathrm{mC}$ to $5 \mathrm{hmC}$ in early embryos. Nature 486 415-419. (doi:10.1038/nature11093)

Obata Y 2011 Study on the mechanism of maternal imprinting during oocyte growth. Journal of Reproduction and Development 57 1-8. (doi:10.1262/jrd.10-195E)

Obata Y \& Kono T 2002 Maternal primary imprinting is established at a specific time for each gene throughout oocyte growth. Journal of Biological Chemistry 277 5285-5289. (doi:10.1074/jbc.M108586200)

Obata Y, Villemure M, Kono T \& Taketo T 2008 Transmission of $Y$ chromosomes from $\mathrm{XY}$ female mice was made possible by the replacement of cytoplasm during oocyte maturation. PNAS 105 13918-13923. (doi:10.1073/pnas.0802680105)

Park S, Zeidan K, Shin JS \& Taketo T 2011 SRY upregulation of SOX9 is inefficient and delayed, allowing ovarian differentiation, in the B6.Y(TIR) gonad. Differentiation 82 18-27. (doi:10.1016/j.diff.2011.04.007)

Sakai Y, Suetake I, Shinozaki F, Yamashina S \& Tajima S 2004 Co-expression of de novo DNA methyltransferases Dnmt3a2 and Dnmt3L in gonocytes of mouse embryos. Gene Expression Patterns 5 231-237. (doi:10.1016/ j.modgep.2004.07.011)

Schoenherr CJ, Levorse JM \& Tilghman SM 2003 CTCF maintains differential methylation at the Igf $2 / \mathrm{H} 19$ locus. Nature Genetics 33 66-69. (doi:10.1038/ng1057)

Szabo PE, Tang SH, Silva FJ, Tsark WM \& Mann JR 2004 Role of CTCF binding sites in the lgf2/H19 imprinting control region. Molecular and Cellular Biology 24 4791-4800. (doi:10.1128/MCB.24.11.4791-4800. 2004)

Villemure M, Chen HY, Kurokawa M, Fissore RA \& Taketo T 2007 The presence of $\mathrm{X}$ - and $\mathrm{Y}$-chromosomes in oocytes leads to impairment in the progression of the second meiotic division. Developmental Biology 301 1-13. (doi:10.1016/j.ydbio.2006.10.034)

Wang L, Cao J, Ji P, Zhang D, Ma L, Dym M, Yu Z \& Feng L 2012 Oocyte-like cells induced from mouse spermatogonial stem cells. Cell \& Bioscience 2 27. (doi:10.1186/2045-3701-2-27)

Received 6 August 2013

First decision 2 October 2013

Revised manuscript received 6 October 2013

Accepted 8 October 2013 